\title{
Advanced Smart Cruise Control with Safety Distance Considered Road Friction Coefficient
}

\author{
Doui Hong, Chanho Park, Yongho Yoo, and Sungho Hwang
}

\begin{abstract}
This research suggests the velocity controller with advanced smart cruise control (ASCC) with stop \& go control considering the surface friction coefficient and applying a safety distance. A safety distance affects quality of cruise control. If a safety distance is too short, the probability to cause a collision gets greater whereas if it is too long, it can cause traffic congestion. Thus, calculating the optimal safety distance is very important. In this study, we used fitting functions to obtain a basic safety distance and then added the $\mu$-safety distance, considering friction coefficient and relative velocity, to calculate final safety distance. We invented ASCC and stop \& go control by considering velocity of vehicle to maintain the safety distance and relative velocity at the same time. Carsim was used for simulation and we found that while a vehicle with the velocity controller, calculating the velocity and location difference between preceding and following vehicles with four friction coefficients, it keeps a safety distance.
\end{abstract}

Index Terms-Advanced smart cruise control (ASCC), friction coefficient, safety distance.

\section{INTRODUCTION}

Adaptive Cruise control with stop \& go has been highlighted in the field of longitudinal velocity control of vehicle. This system allows vehicles to keep a certain distance and to follow a preceding vehicle by calculating the speed of front preceding vehicles, speed of following vehicles, and the distance between cars. One of important components in Adaptive Cruise control with stop \& go system is calculating a safety distance. Many researchers calculated it by multiplying the speed of vehicle and time-constant [1]. Some researchers suggested to add anti-collision constant to the equation [2]-[4]. Deng and Yingping defined the second degree equation of the safety distance according to the speed using fitting function [5]. The safety distance should be the distance preventing collision with preceding vehicle. Breaking distance depends on surface friction coefficient, so that the safety distance should also vary from the surface friction coefficient [6].

In this paper, we derive a basic safety distance formula by

Manuscript received November 30, 2014; revised March 2, 2015. This research was supported by Basic Science Research Program through the National Research Foundation of Korea (NRF) and funded by the Ministry of Education (NRF-2013R1A1A2005594).

Doui Hong, Chanho Park, and Sungho Hwang are with School of Mechanical Engineering, Sungkyunkwan University, Suwon, 440-746, Republic of Korea (e-mail: hdo4236@gmail.com, pch881125@gmail.com, hsh@me.skku.ac.kr).

Yongho Yoo is with the Korea Automotive Technology Institute, 303 Pungse-ro, Pungse-myeon, Dongnam-gu, Cheonan-si, Chungnam 330-912, Republic of Korea (e-mail: yhyoo@katech.re.kr). using fitting functions and braking distance data, provided by Road Traffic Authority. Moreover, we obtained $\mu$-safety distance using a friction coefficient of surface and a calculate safety distance of vehicle.

After that, we calculate desired velocity of vehicle sustaining safety distance. According to calculation, after we designed ASCC with stop \& go control, we demonstrated the utility of $\mu$-safety distance through the graphs of vehicle interval and speed and confirmed the performance of ASCC with stop \& go control.

\section{Calculation OF SAFETy Distance}

In this study, we obtained a basic safety distance using fitting function and add $\mu$-safety distance, considering a friction coefficient and a relative velocity, to the results to calculate a final safety distance.

\section{A. Calculation of Basic Safety Distance}

In this research, a basic safety distance means a braking distance of vehicle in usual road condition. Provided braking distance data by Road Traffic Authority of Korea was used because braking power varied from types of vehicle when general vehicles brake suddenly. A basic safety distance can be obtained by applying fitting function to previous data. Through this progress, basic safety distance $L_{b}$ can be expressed as follows.

$$
L_{b}=a_{1}+a_{2} v+a_{3} v^{2}
$$

In the above equation, where $v$ is the speed of vehicle, $a_{1}$ means the minimum maintained distance when two vehicles stop and $a_{1}, a_{2}$, and $a_{3}$ are all constants.

\section{B. Calculation of $\mu$-Safety Distance}

In practical environment, friction coefficient between road and tire vary from environment. In general, a friction coefficient of dry road is 0.8 , that of wet road is 0.5 , that of snowy road is 0.3 , and that of icy road is 0.2 [7]. A friction coefficient makes a braking distance to differ. Thus, a friction coefficient is absolutely necessary when a safety distance is calculated. In this study, after obtaining a friction coefficient using parameter, which can be obtained only in vehicle, we calculated $\mu$-safety distance by using the results.

To derive a simple equation, we only considered drag resistance and ignored rolling resistance and gradient resistance to calculate. Here, driving force is calculated by multiplying normal force and friction coefficient, assuming that a front wheel drive car accelerates. 


$$
\begin{aligned}
F & =m a \\
& =\text { driving force }- \text { drag force } \\
& =\left(-\mu m g \frac{h}{l}+m g \frac{l_{r}}{l}\right) \mu-\frac{1}{2} C_{w} A \rho v^{2}
\end{aligned}
$$

If we derive the equation for $\mu$ from this equation, it can be expressed as equation (3).

$$
\mu^{2} m g \frac{h}{l}-\mu m g \frac{l_{r}}{l}+C_{w} A \frac{1}{2} \rho v^{2}+m a=0
$$

Thus, a final equation is derived as follows.

$$
\mu=\frac{m g \frac{l_{r}}{l} \pm \sqrt{\left(m g \frac{l_{r}}{l}\right)^{2}-4 m g \frac{h}{l}\left(C_{w} A \frac{1}{2} \rho v^{2}+m a\right)}}{2 m g \frac{h}{l}}
$$

where $l$ is a distance between the center of a front wheel and the center of a rear wheel, $l_{f}$ is a distance between the center of vehicle and the center of a front wheel, $l_{r}$ is a distance between the center of vehicle and the center of a rear wheel, and $h$ is a height from the ground to the center of vehicle height. Also, where $\rho$ means air density, $C_{w}$ means drag coefficient, $A$ means the front part area of vehicle, $m$ is vehicle weight, $a$ is the acceleration of vehicle, and $g$ is acceleration of gravity.

Following graph is $\mu$-slip curve by calculating equation (4).

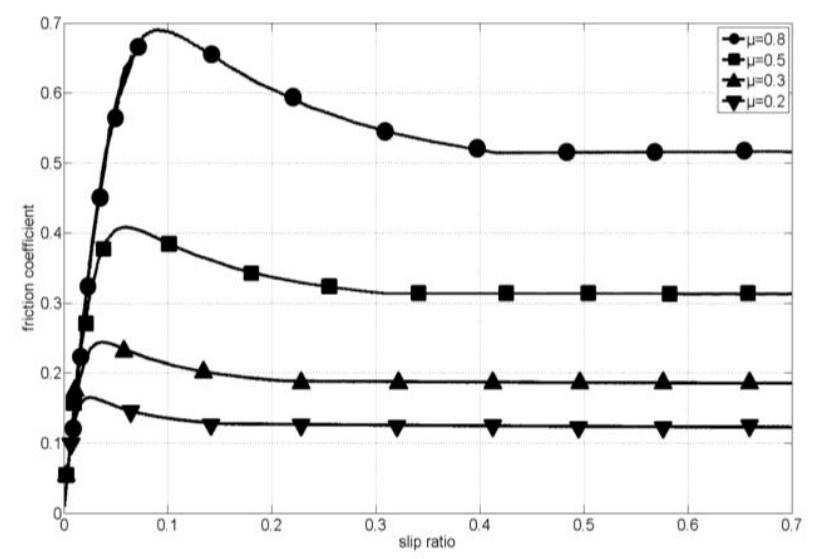

Fig. 1. $\mu$-slip curve.

According to this, we assumed a friction coefficient and a calculated $\mu$-safety distance. When a moving object with a certain speed $v_{0}$ decelerates with a certain acceleration $a$, the distance that the object moves and the speed $v$ are as follows.

$$
\begin{gathered}
L_{0}=v_{0} t+1 / 2 \cdot a t^{2} \\
v=v_{0} t+a t
\end{gathered}
$$

Here, acceleration $a$ is $\mu g$, so that $\mu$-safety distance $L_{m}$ is expressed as a following equation.

$$
L_{m}=\frac{v^{2}-v_{0}^{2}}{2 \mu g}
$$

Therefore, the safety distance $L_{s}$ is finally expressed as follows.

$$
\begin{aligned}
L_{s} & =L_{b}+L_{m} \\
& =\left(a_{1}+a_{2} v_{1}+a_{3} v_{1}^{2}\right)+\frac{v_{1}^{2}-v_{2}^{2}}{2 \mu g}
\end{aligned}
$$

\section{VELOCITY CONTROL}

If a safety distance between a preceding vehicle and a following vehicle is given in Adaptive Cruise Control, a essential control component is a required velocity which can maintain a given safety distance. We used various components such as preceding vehicle velocity $v_{1}$, following vehicle velocity $v_{2}$ and a distance from preceding vehicle $L$ to obtain proper velocity. The speed plan can be obtained by considering the ratio of a distance from preceding vehicle $L$ and a safety distance $L_{s}$. Briefly, we calculate the speed plan by calculating the sum of both a velocity component of maintaining certain distance with preceding vehicle $v_{\text {following }}$ and a velocity component $v_{\text {relative }}$ which considers $L_{s}$ and relative velocity. When velocity $v$ decreases linearly depending on a distance with preceding vehicles, a moving distance by time $s$ can be defined as equation (1).

$$
\begin{gathered}
s=L-L_{s} \\
v=\frac{d s}{d t}=k s \\
\frac{d s}{s}=k d t \\
s=e^{k t+s_{0}}, v=k e^{k t+s_{0}}, a=k^{2} e^{k t+s_{0}}
\end{gathered}
$$

$k$ means a negative constant. It was found that by means of this equation, the velocity and acceleration decrease as vehicle attains a safety distance. In case when $s<0$, it means that a following vehicle can approach the preceding car across a safety distance. Thus, to prevent a collision, a velocity of the following car is required to decrease rapidly by driving a squaring distance that is between a preceding car and the following car. Squaring a decelerating velocity is different from the conventional calculation. Therefore, the moving distance $s$ by time can be obtained as follows.

$$
\begin{gathered}
v=-k \sqrt{-s} \\
\frac{d s}{\sqrt{-s}}=k d t \\
s=-\frac{1}{4}\left(s_{0}-k t\right)^{2}
\end{gathered}
$$

Unlike the above equation, it is found that variation of moving distance gets greater and the distance between vehicles gets far rapidly as time goes. 
To derive equation of velocity, if the distance $L$ with preceding car gets closer to $L_{s}$, the velocity of vehicle changes linearly with the velocity of preceding vehicle and when it becomes that $L=L_{s}$, the desired velocity of vehicle $v_{d}$ should equal to $v_{1}$. Moreover, considering a relative velocity, a safety distance $L_{s}$, and a distance $L$ between a preceding vehicle and a following vehicle, the vehicle should maintain the given safety distance at the same speed of preceding vehicle by additional accelerations and decelerations. Further, when $L<L_{s}$, it should decelerate rapidly and prevent a collision with preceding car. We suggest the equation of speed control which satisfies these conditions as follows.

$$
\begin{aligned}
v_{d} & =\frac{L-L_{s}}{L_{s}}\left(\left|v_{1}-v_{2}\right|\right)+\frac{L}{L_{s}} v_{1}, \quad L-L_{s} \geq 0 \\
& =\frac{L-L_{s}}{L_{s}}\left(\left|v_{1}-v_{2}\right|\right)+\left(\frac{L}{L_{s}}\right)^{2} v_{1}, L-L_{s}<0
\end{aligned}
$$

\section{Simulation AND Results}

TABLE I: PARAMETERS OF SIMULATION
\begin{tabular}{|c|c|}
\hline Drag coefficient & 0.35 \\
\hline Air density & $1.206 \mathrm{~kg} / \mathrm{m}^{3}$ \\
\hline Vehicle cross section areav & $1.6 \mathrm{~m}^{2}$ \\
\hline Center of Gravity height & $540 \mathrm{~mm}$ \\
\hline Wheel base & $2978 \mathrm{~mm}$ \\
\hline Longitudinal position of front wheel from vehicle CG & $1016 \mathrm{~mm}$ \\
\hline Longitudinal position of rear wheel from vehicle CG & $1562 \mathrm{~mm}$ \\
\hline Wheel diameter & $620 \mathrm{~mm}$ \\
\hline Vehicle mass & $1274 \mathrm{~kg}$ \\
\hline
\end{tabular}

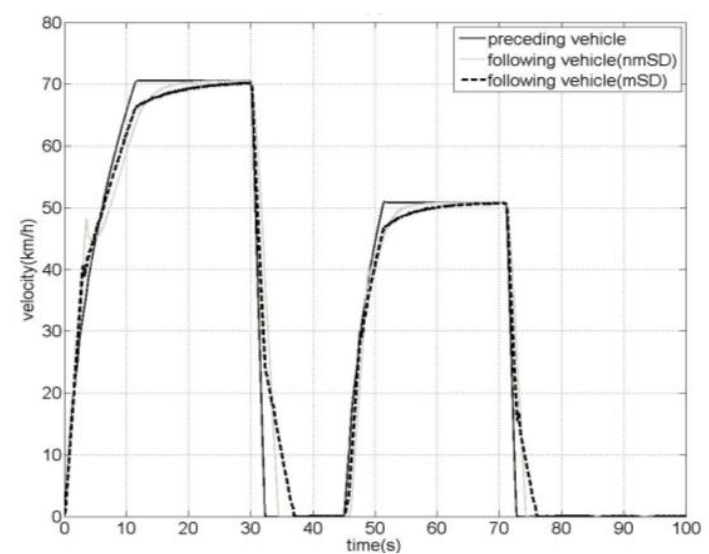

(a)

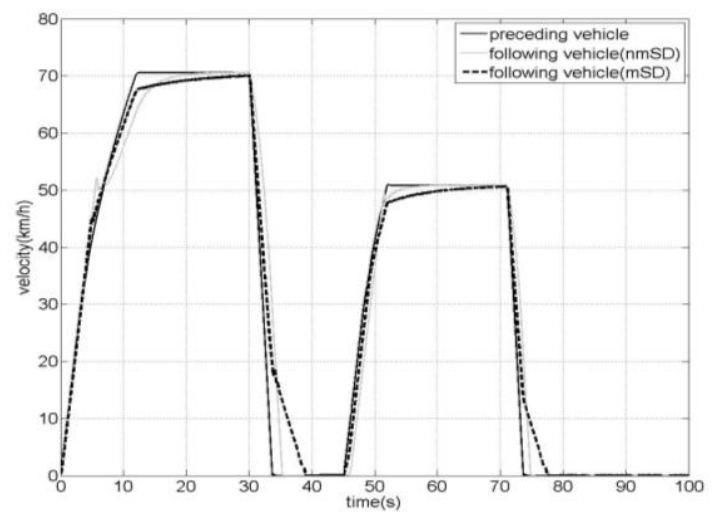

(b)

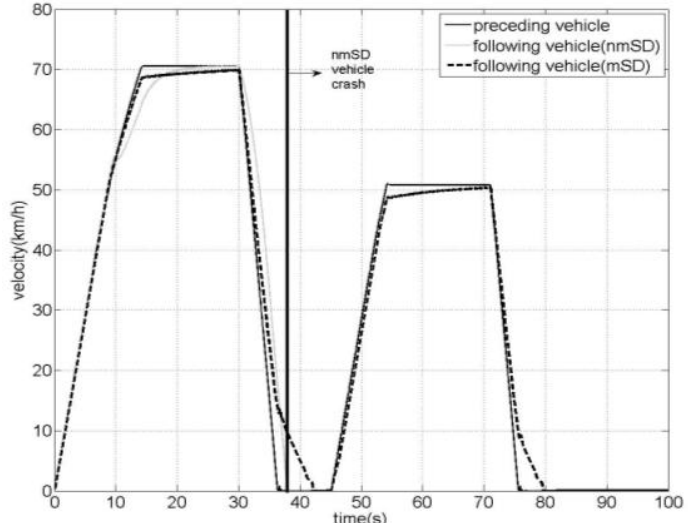

(c)

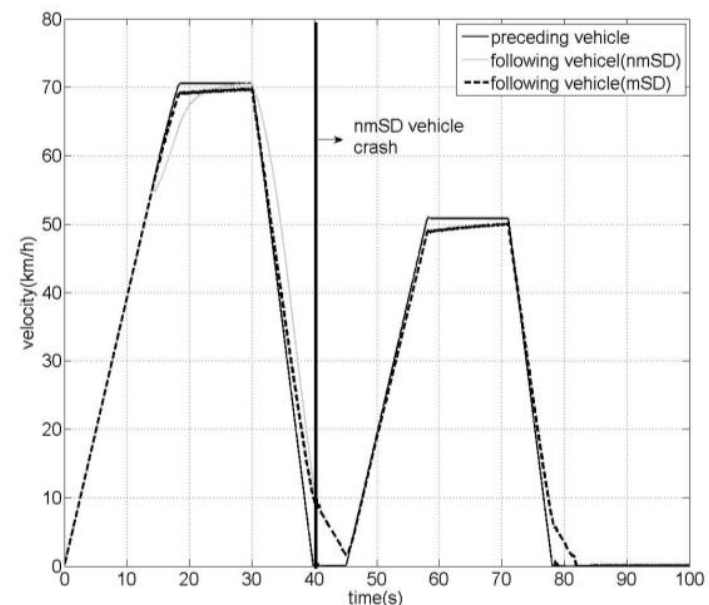

(d)

Fig. 2. Time-velocity graph. (a) $\mu=0.8$. (b) $\mu=0.5$. (c) $\mu=0.3$.(d) $\mu=0$.2. mSD (with $\mu$-safety distance), nmSD (without $\mu$-safety distance).

To verify equation for a safety distance and a velocity controller we suggested, we performed simulation using Carsim and specified parameter as follows.

As initial conditions, initial velocities of preceding vehicle and following vehicle are $0 \mathrm{~km} / \mathrm{h}$, and a distance between vehicles is $20 \mathrm{~m}$. Assuming the situation that the preceding car accelerated up to $70 \mathrm{~km} / \mathrm{h}$, it braked suddenly, and it accelerated up to $50 \mathrm{~km} / \mathrm{h}$ and again it braked suddenly. In this case, we presumed that the delay time when the following vehicle received the location of preceding vehicle, calculated and controlled its speed is $0.3 \mathrm{~s}$. We carried out the simulation by assuming various road conditions (e.g. dry $(\mu=0.8)$, wet $(\mu=0.5)$, snowy $(\mu=0.3)$, icy $(\mu=0.2))$ and observed motions of following vehicle. A vehicle, which $\mu$-safety distance was applied, is called as mSD and the other vhicle without $\mu$ -safety distance system is called as nmSD. The minimum safety distance $a_{1}$ was set as $1.5 \mathrm{~m}$. The velocity algorithm of each following car was performed equally.

Fig. 2 shows the speed of preceding vehicle, the speed of following vehicle with the $\mu$-safety distance system, and the speed of following vehicle without the $\mu$-safety distance system. In case of the vehicle, which $\mu$-safety distance is applied, it was observed that it smoothly approached to the preceding car and manipulated its speed to be equal to the preceding car, compared to the other vehicle. In case of the vehicle without the $\mu$-safety distance system, when it approaches the stopped car, it urgently slows down and stops. On the other hand, when the car with $\mu$-safety distance system 
approached the preceding car, it approached smoothly with low velocity. In Fig. 2(c) and (d), speed data of the vehicle without $\mu$-safety distance system after collision isn't plotted in the graph because it collided with the preceding car.

As shown in Fig. 3, the changes of distance between a preceding vehicle and a following vehicle under the various circumstances are plotted in the graphs. In case of Fig. 3(c) and (d), it shows that the vehicle without the $\mu$-safety distance system has a minus distance between preceding and following cars, so that it presents that the following one crashed with the preceding one. Further, if $\mu$-safety distance system is applied, the safety distance between preceding and following vehicles varies from each friction coefficient whereas if it is not applied, the safety distance comes out same even under various friction coefficients.

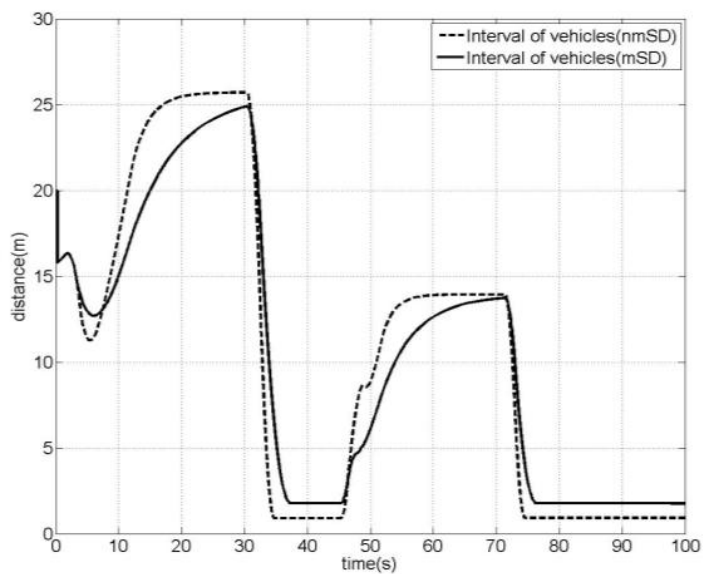

(a)

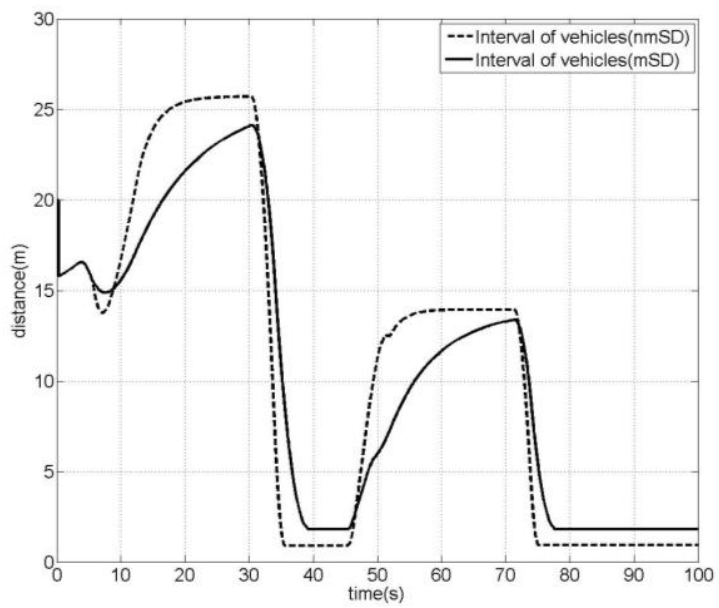

(b)

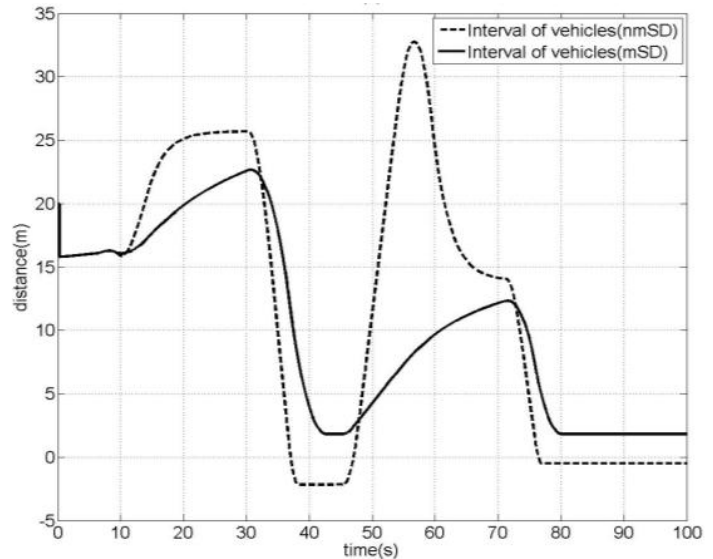

(c)

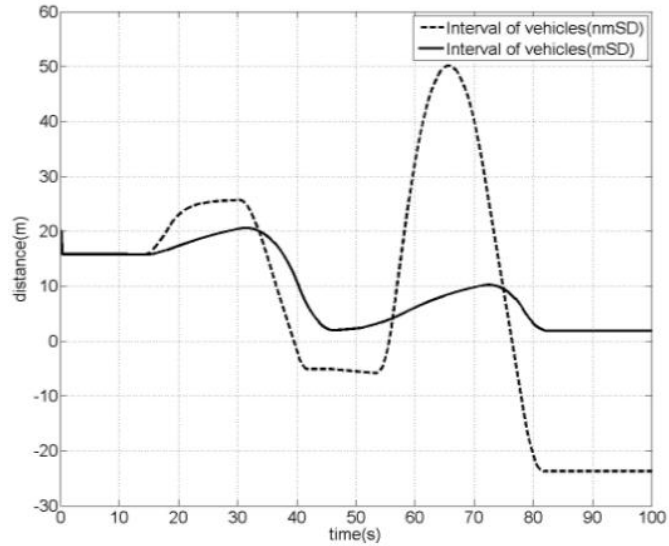

(d)

Fig. 3. Time-distance graph (a) $\mu=0.8$. (b) $\mu=0.5$. (c) $\mu=0.3$. (d) $\mu=0.2$. mSD (with $\mu$-safety distance), $\mathrm{nmSD}$ (without $\mu$-safety distance).

\section{CONCLUSION}

In this paper, we calculated the safety distance by considering the friction coefficient and derived the equation of a required velocity, thus it allows vehicles to go as it maintains the safety distance. Also, we demonstrated that the given safety distance is necessary through simulations and observed the vehicle with speed plan runs as it keep the safety distance. Especially, it is found that if a vehicle with $\mu$-safety distance system runs, it can avoid collision, maintain the safety distance and follow the preceding vehicle even under unfavorable road conditions with low friction coefficient. The smooth driving reduces driver's anxiety and further it helps driver to drive vehicle stably under rainy and snowy conditions. In short, this technique is expected to raise convenience of drivers in every condition.

\section{REFERENCES}

[1] Z. Ali, A. A. Popov, and G. Charles, "Model predictive control with constraints for a nonlinear adaptive cruise control vehicle model in transition manoeuvres," Vehicle System Dynamics, vol. 51, no. 6, pp. 943-963, 2013.

[2] S. Oncu et al., "Cooperative adaptive cruise control: Network-aware analysis of string stability," Browse Journals \& Magazines, vol. 15, issue 4.

[3] C. C. Tsai, S. M. Hsieh, and C. T. Chen, "Fuzzy longitudinal controller design and experimentation for adaptive cruise control and stop\&go," Journal of Intelligent \& Robotic Systems, vol. 59, no. 2, pp. 167-189, 2010.

[4] P. Shakouri, A. Ordys, and M. R. Askari, "Adaptive cruise control with stop\&go function using the state-dependent nonlinear model predictive control approach," ISA Transactions, vol. 51, no. 5, pp. 622-631, 2012.

[5] P. Deng and Y. P. Zheng, "Velocity difference control based on dynamic tracking of safe following distance in the process of vehicle following," Intelligent Transport Systems, vol. 8, no. 3, pp. 232-243, 2014.

[6] S. W. Moon, I. Moon, and K. S. Yi, "Design, tuning, and evaluation of a full-range adaptive cruise control system with collision avoidance," Control Engineering Practice, vol. 17, no. 4, pp. 442-455, 2009.

[7] C.-Y. Lee, K. Hedrick, and K.-S. Yi, "Real-time slip-based estimation of maximum tire-road friction coefficient," IEEE/Asme Transactions on Mechatronics, vol. 9, no. 2, pp. 454-458, 2004.

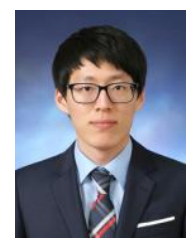

Doui Hong received the B.S. degree in mechanical engineering from Sungkyunkwan University, Suwon, Korea in 2014. He is currently working toward the M.S degree with the School of Mechanical Engineering, Sungkyunkwan University, Suwon, Korea. His research interests include the driverless vehicles and vehicle dynamics. 


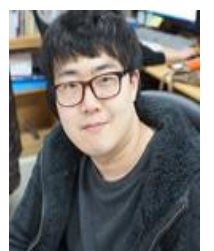

Chanho Park received the B.S. degrees in mechanical engineering from Sungkyunkwan University, Suwon, Korea, in 2014. He is currently working toward the M.S degree with the School of Mechanical Engineering, Sungkyunkwan University, Suwon, Korea. His research interests include the driverless vehicles and vehicle dynamics.

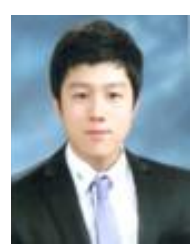

Yongho Yoo received the B.S. degree in automotive engineering from Kongju National University, Cheonan, Republic of Korea in 2011 and he is currently working for a M.S. degree in mechatronics engineering at Sungkyunkwan University, Suwon, Republic of Korea. His research interests are vehicle dynamics, vehicle stability control and tyre wet grip for tyre labeling system.

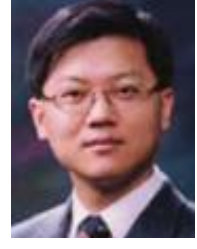

Sungho Hwang received the B.S. degree in mechanical design and production engineering and the M.S. and $\mathrm{Ph} . \mathrm{D}$. degrees in mechanical engineering from Seou National University, Seoul, Korea, in 1988, 1990, and 1997 , respectively. He is currently an associate professor with the School of Mechanical Engineering, Sungkyunkwan University, Suwon, Korea. 